\title{
Cervical Dorsal Rhizotomy Enhances Serotonergic Innervation of Phrenic Motoneurons and Serotonin-Dependent Long-Term Facilitation of Respiratory Motor Output in Rats
}

\author{
Richard Kinkead, ${ }^{3}$ Wen-Zhi Zhan, ${ }^{2}$ Y. S. Prakash, ${ }^{2}$ Karen B. Bach, ${ }^{1}$ Gary C. Sieck, ${ }^{2}$ and Gordon S. Mitchell \\ ${ }^{1}$ Department of Comparative Biosciences, School of Veterinary Medicine, University of Wisconsin, Madison, Wisconsin \\ 53706, 2Department of Anesthesiology and Department of Physiology and Biophysics, Mayo Clinic, Rochester, \\ Minnesota 55905, and 3Unité de Recherche en Pédiatrie, Centre Hôspitalier Universitaire de Québec, \\ Pavillon St-François d'Assise, Québec, QC G1L 3L5 Canada
}

We tested the hypothesis that spinal plasticity elicited by chronic bilateral cervical dorsal rhizotomy $\left(\mathrm{C}_{3}-\mathrm{C}_{5}\right.$; $\left.\mathrm{CDR}\right)$ has functional implications for respiratory motor control. Surgery was performed on rats (CDR or sham-operated) $26 \mathrm{~d}$ before phrenic motoneurons were retrogradely labeled with cholera toxin. Rats were killed $2 \mathrm{~d}$ later, and their spinal cords were harvested and processed to reveal the cholera toxin-labeled phrenic motoneurons and serotonin-immunoreactive terminals. The number of serotonin-immunoreactive terminals within $5 \mu \mathrm{m}$ of labeled phrenic motoneuron soma and primary dendrites increased 2.1-fold after CDR versus sham-operation. Timedependent phrenic motor responses to hypoxia were compared among CDR, sham-operated, and control rats. Anesthetized, paralyzed, vagotomized, and artificially ventilated rats were exposed to three, 5 min episodes of isocapnic hypoxia $\left(\mathrm{Fi}_{\mathrm{O}_{2}}=\right.$ $0.11)$, separated by 5 min hyperoxic intervals $\left(\mathrm{Fi}_{\mathrm{O}_{2}}=0.5\right)$. One hour after hypoxia, a long-lasting, serotonin-dependent enhancement of phrenic motor output (long-term facilitation) was observed in both sham and control rats. After CDR, long-term facilitation was 108 and $163 \%$ greater than control and sham responses, respectively. Pretreatment of CDR rats with a $5-\mathrm{HT}_{2}$ receptor antagonist (ketanserin tartrate, $2 \mathrm{mg} / \mathrm{kg}$, i.v.) before episodic hypoxia prevented long-term facilitation and revealed a modest $(-28 \pm 13 \% ; p<0.05)$ long-lasting depression of phrenic motor output. The results indicate that CDR: (1) increases serotonergic innervation of the phrenic motor nucleus; and (2) augments serotonin-dependent long-term facilitation of phrenic motor output. These results further suggest a form of plasticity based on changes in the capacity for neuromodulation.

Key words: plasticity; serotonin; respiratory control; longterm facilitation; rhizotomy; phrenic motoneurons
Descending serotonergic pathways can undergo significant plasticity (Azmitia and Whitaker-Azmitia, 1991; Whitaker-Azmitia and Azmitia, 1991). For instance, spinal sensory denervation by dorsal rhizotomy increases serotonin immunoreactivity in the dorsal horn in rats (Marlier et al., 1991a; Zhang et al., 1993). However, relatively little is known concerning the effects of chronic deafferentation on serotonergic innervation of the spinal ventral horn. Studies in goats indicate that serotonergic immunoreactivity is increased in the ventral horn of thoracic spinal segments after bilateral thoracic dorsal rhizotomy (Mitchell et al., 1992). Thoracic dorsal rhizotomy also increases serotonin concentration in cervical spinal segments associated with the phrenic motor nucleus in goats $\left(\mathrm{C}_{5}-\mathrm{C}_{7}\right)$, a site distant from the dorsal root transection (Mitchell et al., 1995; Turner et al., 1997). It was suggested that an upregulation of the serotonergic system in the thoracic and cervical spinal cord enhances phrenic and intercostal motoneuron excitability, thereby compensating for the functional deficits in respiratory motor control caused by thoracic dorsal rhizotomy (McCrimmon et al., 1995; Turner et al., 1997). How-

\footnotetext{
Received June 8, 1998; revised Aug. 3, 1998; accepted Aug. 7, 1998.

This research was supported by National Institutes of Health Grants HL36780, HL53319, HL34817, and HL37680, and by fellowships from Abbott Laboratories (Y.S.P.) and the Medical Research Council of Canada (R.K.). The authors would like to thank Mr. Philip Zhan for his technical support.

Correspondence should be addressed to Dr. Gordon Mitchell, Department of Comparative Biosciences, School of Veterinary Medicine, 2015 Linden Drive West, Madison, WI 53706.

Copyright (C) 1998 Society for Neuroscience $\quad 0270-6474 / 98 / 208436-08 \$ 05.00 / 0$
}

ever, the functional significance of upregulation in spinal serotonin after dorsal rhizotomy remains unclear because a causal relationship is yet to be established. Thus, the primary objective of this study was to develop a model allowing study of the functional consequences of enhanced serotonergic innervation of the spinal cord after chronic deafferentation.

One specific example of serotonin-dependent plasticity in a respiratory motor behavior is long-term facilitation (LTF) of respiratory motor output (Eldridge and Millhorn, 1986; McCrimmon et al., 1995). Long-term facilitation of inspiratory nerve activity is elicited by episodic stimulation of chemosensory afferent pathways, either by electrical (Millhorn et al., 1980a, b; Eldrige and Millhorn, 1986; Hayashi et al., 1993; Fregosi and Mitchell, 1994) or hypoxic stimulation of carotid chemoafferent neurons (Bach and Mitchell, 1996a). This long-lasting (hours) enhancement of respiratory motor output requires serotonin receptor activation because cats or rats pretreated with certain serotonin receptor antagonists (methysergide, ketanserin) fail to develop long-term facilitation (Eldrige and Millhorn, 1986; Bach and Mitchell, 1996a; R. Kinkead and G. Mitchell, unpublished observations). It has been suggested that repeated stimulation of carotid chemoafferent pathways enhances serotonin release in the vicinity of respiratory motoneurons, thereby augmenting their excitability and resulting in long-term facilitation (McCrimmon et al., 1995).

In this study, we investigated the effects of spinal plasticity in descending serotonergic innervation on a serotonin-dependent 
respiratory motor behavior, long-term facilitation. Our working hypothesis was that cervical dorsal rhizotomy $\left(\mathrm{C}_{3}-\mathrm{C}_{5}\right)$, thereby removing primary sensory afferent inputs in the vicinity of the phrenic motor nucleus, would: (1) increase the number of immunoreactive serotonin terminals in the immediate vicinity of phrenic motoneurons retrogradely labeled with cholera toxin B-fragment and (2) enhance serotonin-dependent long-term facilitation of phrenic nerve activity after episodic hypoxia.

Some of these results have been reported previously in abstract form (Kinkead et al., 1997; Prakash et al., 1997).

\section{MATERIALS AND METHODS}

Experiments were performed on 52 adult male rats (Harlan Sprague Dawley, Madison, WI) ranging in mass from 320-523 gm (mean, $438 \pm$ $46 \mathrm{gm}, \mathrm{SD}$ ). The study involved two series of experiments. The first series addressed the effects of chronic bilateral cervical dorsal rhizotomy $\left(\mathrm{CDR} ; \mathrm{C}_{3}-\mathrm{C}_{5}\right)$ on serotonergic innervation of the phrenic motor nucleus. This series involved three groups of rats: bilateral CDR $(n=6)$, shamoperated $(n=6)$, and unoperated controls $(n=2)$. The second series of experiments addressed the functional implications of chronic CDR by comparing short- and long-term changes in inspiratory motor output, during and after exposure to episodic hypoxia. Five groups of animals were studied: $\mathrm{CDR}(n=6)$, CDR pretreated with the $5-\mathrm{HT}_{2}$ receptor antagonist ketanserin $(n=6)$, sham-operated $(n=6)$, unoperated hypoxic control $(n=13)$, and unoperated hyperoxic control rats $(n=7)$. All procedures were approved by the University of Wisconsin animal care and use committee.

Surgical procedures. Bilateral CDR and sham operations were performed under deep sodium pentobarbital $(65 \mathrm{mg} / \mathrm{kg}$, i.p.) anesthesia after anesthetic induction with isoflurane. Supplemental pentobarbital injections were given as necessary. A dorsal incision was made, and the neck muscles were separated and retracted to expose the cervical vertebrae. Laminectomy was then performed on cervical vertebrae $2-5$, and the exposed dura was cut. Using a Zeiss operating microscope, the dorsal roots were separated from the arachnoid and as many radicular vessels as possible. The rootlets were then cut from $\mathrm{C}_{3}$ to $\mathrm{C}_{5}$ with fine scissors. An equal number of sham surgeries were performed in which the dorsal roots were isolated but not cut. The muscles were sutured in anatomical layers, and the skin was closed. The front and hind claws were trimmed to minimize injury caused by scratching after surgery. Rats were allowed to recover for $28 \mathrm{~d}$ before being killed to harvest the spinal cord, or subjected to an acute neurophysiological protocol. Recovery was uneventful in all cases; scratching of the wound was minimal and there were no signs of autotomy.

Estimation of serotonergic innervation to the phrenic motor nucleus. Twenty six days after surgery, phrenic motoneurons were retrogradely labeled with cholera toxin B-fragment injected into the diaphragm of CDR, sham-operated, and control rats. Rats were anesthetized with an intramuscular injection of ketamine and xylazine $(70$ and $7 \mathrm{mg} / \mathrm{kg}$, respectively) after anesthetic induction with isoflurane. Once the animals had reached a surgical plane of anesthesia, two lateral incisions were made below the rib cage, and the skin was retracted to expose the diaphragm. Five to eight intramuscular injections of a $0.5 \%$ cholera toxin B fragment (List Biologicals) in saline containing 1\% Evans blue were made on each side with a $10 \mu$ l Hamilton syringe fitted with a pulled glass pipette. The total volume injected ranged between 35 and $40 \mu \mathrm{l}$ on each side. Two days later, the rats were deeply anesthetized with sodium pentobarbital $(75 \mathrm{mg} / \mathrm{kg}$, i.p.; after anesthetic induction with isoflurane) for transcardiac perfusion with heparinized saline followed by $4 \%$ paraformaldehyde solution in $0.1 \mathrm{M}$ phosphate buffer. The spinal cord was excised and immersed in $4 \%$ paraformaldehyde overnight. The tissue was then transferred to a $25 \%$ sucrose solution before sectioning on a cryostat. Coronal sections of the cervical spinal cord were cut at $50 \mu \mathrm{m}$, and phrenic motoneurons were detected by immunofluorescence using a goat anti-cholera toxin B primary antibody (List Biologicals; 1:10,000) and a Cy3-conjugated anti-goat secondary antibody (Jackson ImmunoResearch, West Grove, PA; 1:1,000). Serotonin terminals were detected by immunofluorescence using rabbit antibody to serotonin (Incstar) and a Cy-5-conjugated anti-rabbit secondary antibody (Jackson ImmunoResearch).

Phrenic motoneurons were sampled along $\mathrm{C}_{3}-\mathrm{C}_{5}$ using the Cavalieri principle. In essence, starting with a randomly selected motoneuron in the $\mathrm{C}_{3}$ segment, every tenth labeled motoneuron was sampled in the rostrocaudal direction. Within each spinal cord section, only whole motoneurons with visible nuclei that were not damaged by the sectioning process were sampled. Using this procedure, 22-25 motoneurons per animal were sampled along the phrenic motor column.

A Bio-Rad MRC500/600 confocal microscope equipped with an Ar-Kr laser was used to simultaneously visualize phrenic motoneurons and serotonergic terminals. A $40 \times / 1.3$ NA objective lens was used for all imaging. Image size was $397 \times 512$ pixels for each of Cy3- and Cy5labeled structures. Optical sections were obtained by focusing at $0.8 \mu \mathrm{m}$ intervals through the depth of the tissue section, matching the section thickness of the $40 \times$ objective lens (Prakash et al., 1993).

The images were reconstructed in three dimensions and analyzed using a comprehensive image manipulation and analysis software package (ANALYZE, Mayo Biomedical Imaging Resources). In this analysis, if a motoneuron structure is present, then the number of immunoreactive terminals was counted. To estimate serotonergic terminal numbers, proximity to phrenic motoneurons was arbitrarily defined at 5 $\mu \mathrm{m}$ from the soma or primary dendrites. It was beyond the scope of the present study to quantify serotonergic terminals in the vicinity of secondary and higher order phrenic motoneuron dendrites. A stereological technique was again used to count the number of serotonergic terminals conforming to these criteria. Using a $25 \times 25$ pixel grid $(10 \times 10 \mu \mathrm{m}$ grid), somal and dendritic parts of a selected motoneuron (see above) were located within systematically sampled grid squares, and the number of serotonergic terminals within $5 \mu \mathrm{m}(12 \times 12$ pixel grid or a quarter of the larger grid) was counted using a blind method. Using this approach, a "spot" of Cy5 immunoreactivity constituted a single 5-HT terminal; larger clusters of immunoreactivity were counted as one terminal. The total number of terminals was then determined by extrapolating the results to the total number of grid squares containing any somal or primary dendritic portion of the selected motoneuron. The number of serotonergic terminals was then expressed per motoneuron.

The possibility that motoneuron size was changed by rhizotomy was assessed by measuring the long and short dimensions of motoneuron somata. A more detailed morphometric assessment of motoneuron size was beyond the scope of the present study.

Assessment of long-term facilitation. The methods used to assess the neural correlates of respiratory activity have been described in detail by Bach and Mitchell (1996a). Briefly, rats were initially anesthetized with isoflurane $\left(2.5-3.0 \%\right.$ in $50 \% \mathrm{O}_{2}$, balance $\mathrm{N}_{2}$ ) and then slowly converted to urethane anesthesia $(1.6 \mathrm{gm} / \mathrm{kg}$, i.v. $)$ over a period of $15-20 \mathrm{~min}$. The plane of anesthesia was assessed regularly by testing corneal reflexes and blood pressure responses to toe pinch. One hour after induction of anesthesia, a constant intravenous infusion of a sodium bicarbonate $(5.0 \%)$ and lactated Ringer's solution $\left(50: 50,1.7 \mathrm{ml} \cdot \mathrm{kg}^{-1} \cdot \mathrm{hr}^{-1}\right)$ was initiated to maintain fluid and acid-base balance.

All rats were prepared with a tracheostomy for artificial ventilation (rodent respirator, model 683; Harvard Apparatus, Inc, South Natick, MA) and tracheal pressure measurement (Statham pressure transducer, P23-id). The lungs were hyperinflated roughly once per hour to prevent alveolar atelectasis. The rats were vagotomized bilaterally in the midcervical region and then paralyzed (pancuronium bromide, $2.5 \mathrm{mg} / \mathrm{kg}$, i.v.) to prevent entrainment of respiratory motor output with the ventilator and spontaneous breathing efforts, respectively. End-tidal $\mathrm{CO}_{2}$ was monitored with a flow-through capnograph (Novametrix, Wallingford, CT) with sufficient response time $(<75 \mathrm{msec})$ to measure rat end-tidal $\mathrm{P}_{\mathrm{CO}_{2}}$. Values obtained from this capnograph closely approximated arterial $\mathrm{P}_{\mathrm{CO}_{2}}$ (usually within 1-2 mmHg). Blood samples of $0.3 \mathrm{ml}$ were drawn from a catheterized femoral artery into a $0.5 \mathrm{ml}$ heparinized glass syringe to determine blood gases and pH (ABL-330; Radiometer, Copenhagen, Denmark); unused blood was returned to the animal. Blood gas and $\mathrm{pH}$ values were corrected to the measured rectal temperature of the rat for each sample. Blood pressure was monitored in the femoral artery (Statham pressure transducer, P23-id). Rectal temperature was maintained between 37 and $38^{\circ} \mathrm{C}$ with a heating pad.

Phrenic nerve was isolated unilaterally, using a left dorsal approach, cut distally and desheathed. The nerve was submerged in mineral oil and placed on a bipolar silver recording electrode. Nerve activity was amplified (gain $=10 \mathrm{~K}$; CWE BMA-931 Bioamp, Ardmore, PA), band-pass filtered, $(100 \mathrm{~Hz}-5 \mathrm{KHz})$ and fed to a moving averager (CWE MA-1000; time constant $100 \mathrm{msec}$ ) before being digitized, recorded, and analyzed with computer software developed in our laboratory.

Experimental protocol. Once the rat preparation was ready for experimentation, $60 \mathrm{~min}$ were allowed for the electroneurogram and arterial blood pressure to stabilize under normocapnic $\left(\mathrm{Pa}_{\mathrm{CO}_{2}}=2-3 \mathrm{mmHg}\right.$ 
Table 1. Arterial $\mathrm{P}_{\mathrm{CO}_{2}}$ values measured throughout the long-term facilitation protocol in experimental groups

\begin{tabular}{|c|c|c|c|c|}
\hline $\begin{array}{l}\text { Experimental group } \\
\mathrm{Pa}_{\mathrm{CO}_{2}}(\mathrm{mmHg})\end{array}$ & Baseline & $\begin{array}{l}\text { Hypoxic stimulus } \\
\left(\mathrm{Fi}_{\mathrm{O}_{2}}=0.11\right)\end{array}$ & $\begin{array}{l}30 \text { min post- } \\
\text { hypoxic }\end{array}$ & $\begin{array}{l}60 \text { min post- } \\
\text { hypoxic }\end{array}$ \\
\hline $\begin{array}{l}\text { Hyperoxic control } \\
\quad(n=7)\end{array}$ & $\begin{array}{c}45 \\
(1.4)\end{array}$ & $\begin{array}{c}46 \\
(1.8)\end{array}$ & $\begin{array}{c}45 \\
(1.5)\end{array}$ & $\begin{array}{c}45 \\
(1.5)\end{array}$ \\
\hline $\begin{array}{l}\text { Control } \\
\qquad(n=13)\end{array}$ & $\begin{array}{c}45 \\
(1.0)\end{array}$ & $\begin{array}{l}45.2 \\
(0.9)\end{array}$ & $\begin{array}{c}44 \\
(1.0)\end{array}$ & $\begin{array}{c}45 \\
(1.0)\end{array}$ \\
\hline $\begin{array}{l}\text { Sham-operated } \\
\quad(n=6)\end{array}$ & $\begin{array}{l}42.8 \\
(0.7)\end{array}$ & $\begin{array}{l}45.0^{*} \\
(0.8)\end{array}$ & $\begin{array}{l}42.2 \\
(0.6)\end{array}$ & $\begin{array}{l}43.0 \\
(0.4)\end{array}$ \\
\hline $\begin{array}{l}\text { Bilateral cervical dorsal } \\
\text { rhizotomy }(n=6)\end{array}$ & $\begin{array}{l}42.7 \\
(0.8)\end{array}$ & $\begin{array}{c}44 \\
(1.0)\end{array}$ & $\begin{array}{c}42 \\
(1.0)\end{array}$ & $\begin{array}{l}42.4 \\
(0.7)\end{array}$ \\
\hline $\begin{array}{l}\text { Bilateral cervical dorsal } \\
\text { rhizitomy }+ \text { ketanserin } \\
(n=6)\end{array}$ & $\begin{array}{c}46 \\
(1.2)\end{array}$ & $\begin{array}{c}44 \\
(1.8)\end{array}$ & $\begin{array}{c}46 \\
(1.2)\end{array}$ & $\begin{array}{c}46 \\
(1.2)\end{array}$ \\
\hline
\end{tabular}

$\overline{\text { At all posthypoxic time points, } \mathrm{Pa}_{\mathrm{CO}_{2}} \text { was actively maintained within } 1 \mathrm{mmHg} \text { of the }}$ baseline value or respiratory nerve activities were not included in the analysis. Data presented as means; corresponding SE values are in parentheses.

*Different from baseline $(p<0.05)$.

Table 2. Arterial $\mathbf{P}_{\mathrm{O}_{2}}$ values measured throughout the long-term facilitation protocol in experimental groups

\begin{tabular}{lcccc}
$\begin{array}{l}\text { Experimental group } \mathrm{Pa}_{\mathrm{O}_{2}} \\
(\mathrm{mmHg})\end{array}$ & \multicolumn{4}{c}{ Hypoxic stimulus 30 min post- 60 min post- } \\
\hline Hyperoxic control & 212 & 199 & 210 & 204 \\
$\quad(n=7)$ & $(18)$ & $(8)$ & $(8)$ & $(3)$ \\
Control & 218 & $37^{*}, * * *$ & 210 & 206 \\
$\quad(n=13)$ & $(11)$ & $(1.7)$ & $(12)$ & $(11)$ \\
Sham-operated & 182 & $34^{*}, * * *$ & 179 & $147^{*}, *^{* *}$ \\
$\quad(n=6)$ & $(15)$ & $(1.9)$ & $(13)$ & $(19)$ \\
Bilateral cervical dorsal & 188 & $33^{*}, * * *$ & 181 & 183 \\
$\quad$ rhizotomy $(n=6)$ & $(9.7)$ & $(1.8)$ & $(13)$ & $(13)$ \\
Bilateral cervical dorsal & 236 & $43^{*}, * * *$ & 225 & 230 \\
$\quad$ rhizitomy + ketanserin & $(5.8)$ & $(1.9)$ & $(9.3)$ & $(9.0)$ \\
$\quad(n=6)$ & & & &
\end{tabular}

Hypoxia levels were not significantly different between the four hypoxic groups. Data presented as means, with SEs in parentheses.

*Different from hyperoxic control group $(p<0.05)$.

** Different from control group $(p<0.05)$.

***Different from baseline $(p<0.05)$.

above $\mathrm{CO}_{2}$ apneic threshold; see Table 1) and hyperoxic conditions $\left(\mathrm{Fi}_{\mathrm{O}_{2}}=0.5 ; \mathrm{Pa}_{\mathrm{O}_{2}}>175 \mathrm{mmHg}\right.$, see Table 2$)$. Baseline phrenic nerve activity was achieved by manipulating inspired $\mathrm{CO}_{2}$ and respiratory pump rate and/or volume while monitoring end-tidal $\mathrm{CO}_{2}$ levels until phrenic nerve activity attained low but stable levels of activity. The protocol began with a control arterial blood sample. All subsequent blood gas data were compared with this initial baseline value. Baseline phrenic nerve activity was recorded, followed by three, 5 min episodes of isocapnic hypoxia $\left(\mathrm{Fi}_{\mathrm{O}_{2}}=0.11\right)$ separated by $5 \mathrm{~min}$ of hyperoxia. Table 2 reports the $\mathrm{Pa}_{\mathrm{O}}$, values obtained during the first hypoxic episode in each experimental group.

Although not the focus of the present study, this experimental protocol allowed us to assess two additional aspects of the ventilatory response to hypoxia that occur in a shorter time domain than long-term facilitation (compare Fig. 1): the short-term hypoxic ventilatory response (during hypoxia) and the decline of respiratory frequency immediately after isocapnic hypoxia (posthypoxia frequency decline, PHFD; Hayashi et al., 1993; Coles and Dick, 1996; Powell et al., 1998).

Relative isocapnia was maintained throughout the protocol by monitoring end-tidal $\mathrm{CO}_{2}$ and adjusting inspired $\mathrm{CO}_{2}$ accordingly. Nerve activity was recorded throughout the entire protocol, and a blood sample was drawn during one of the three hypoxic responses at random to assess the level of hypoxia. Blood samples were also taken at both posthypoxic data points (30 and $60 \mathrm{~min}$ ) to assure that arterial $\mathrm{P}_{\mathrm{CO}_{2}}$ was within 1 $\mathrm{mmHg}$ of the baseline value during data collection (Table 1); data that exceeded this blood-gas criterion were not accepted. Therefore, changes in arterial $\mathrm{P}_{\mathrm{CO}_{2}}$ are unlikely to be responsible for long-term facilitation after episodic hypoxia (Bach and Mitchell, 1996a). At the end of the protocol, the response to elevated levels of inspired $\mathrm{CO}_{2}$ (end-tidal $\mathrm{CO}_{2}=90-95 \mathrm{mmHg}$ ) was recorded to obtain a measure of maximal (or at least a standardized hypercapnic control) nerve burst amplitude. To ensure that all changes in phrenic motor output were unrelated to time-dependent changes in blood pressure, anesthesia or other factors, the protocol was repeated in seven rats that were prepared and treated identically to the original experimental animals, but that were never exposed to hypoxia. This group of rats will be referred to as hyperoxic (time) control.

Previous studies in our laboratory have shown that $5-\mathrm{HT}_{2}$ receptor activation is necessary for long-term facilitation (Kinkead and Mitchell, unpublished observations). To confirm that enhanced long-term facilitation of phrenic nerve activity after CDR was related to increased serotonergic function (vs other potential mechanisms), a group of CDR rats received a slow $(\sim 1 \mathrm{~min})$ intravenous injection of ketanserin tartrate (concentration, $2 \mathrm{mg} / \mathrm{ml}$; dose, $2 \mathrm{mg} / \mathrm{kg}$ ), a high affinity $5-\mathrm{HT}_{2}$ receptor antagonist $\left(5-\mathrm{HT}_{2 \mathrm{~A}}, K_{\mathrm{i}}\right.$ range $=0.4-3.1 \mathrm{nM} ; 5-\mathrm{HT}_{2 \mathrm{C}}, K_{\mathrm{i}}$ range $=28-98$ nM; Zifa and Fillion, 1992). In this group, the experiment began when phrenic nerve activity and blood pressure were stable (typically 20-30 min after injection). Baseline phrenic burst amplitude and frequency were not significantly affected by ketanserin $(p=0.154$ and $p=0.300$, respectively).

Data analysis. Peak amplitude and frequency (bursts per minute) of phrenic nerve activity were averaged over a minimum of 50 bursts for each recorded data point. Averaged amplitude data were then normalized as a percentage change from the baseline (prehypoxia) activity and as a change from baseline, expressed as the percentage of the maximum $\left(\mathrm{CO}_{2}\right.$-stimulated $)$ nerve activity. The latter form of normalization obviates concerns about expressing data in terms of the percentage increase above an arbitrary (low) baseline value (cf. Fregosi and Mitchell, 1994). The results were analyzed statistically using a two-way ANOVA (Sigmastat; Jandel Scientific, Corte Madera, CA) followed by pairwise multiple comparisons using Bonferroni's method $(p<0.05)$; a repeated measures design was used when appropriate.

\section{RESULTS}

\section{Series 1: effects of bilateral CDR on serotonergic innervation of the phrenic motor nucleus and motoneuron size}

Figure 2 shows representative micrographs comparing serotonergic innervation of phrenic motoneurons in sham-operated (Fig. $2 A$ ) and CDR rats (Fig. $2 B$ ). Twenty-eight days after surgery, the total number of serotonin-immunoreactive terminals located within $5 \mu \mathrm{m}$ of a given phrenic motoneuron soma (Fig. $3 A$ ) or its primary dendrites (Fig. $3 B$ ) was significantly greater in CDR rats in comparison to sham-operated or control animals (110 and $105 \%$ increase at soma and primary dendrites, respectively). Because the number of serotonin terminals per motoneuron was not significantly different between the unoperated control and sham-operated rats, the data obtained from these two groups were pooled.

Phrenic motoneuron somata were slightly enlarged along the long axis in CDR rats (long axis: sham/control, $43.4 \pm 0.5 \mu \mathrm{m}$ vs CDR, $47.6 \pm 0.9 \mu \mathrm{m}, p<0.05$; short axis: sham/control, $24.0 \pm$ $0.3 \mu \mathrm{m}$ vs CDR, $25.6 \pm 0.4 \mu \mathrm{m}$, not significant).

\section{Series 2: CDR effects on neural correlates of respiration}

The integrated phrenic neurogram shown in Figure 1 demonstrates several time domains associated with the hypoxic phrenic (ventilatory) response. The description of CDR effects on these time-dependent responses will follow in their temporal sequence. 


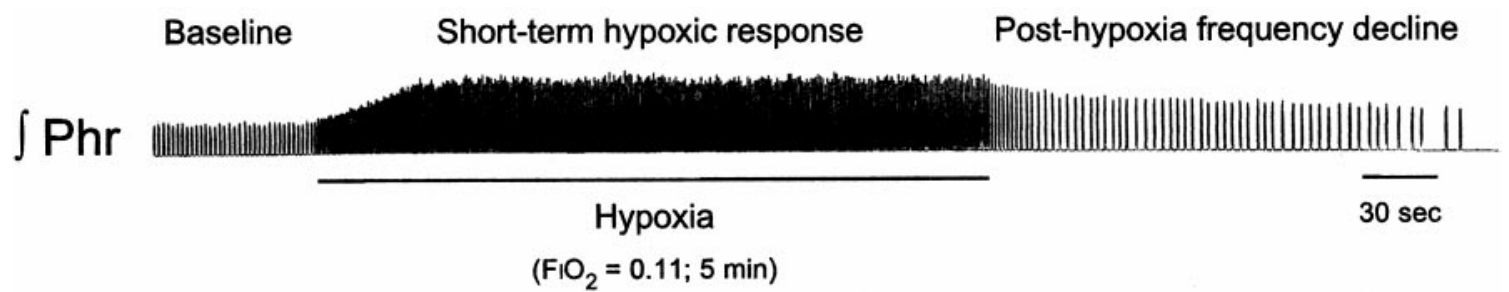

Long-term facilitation

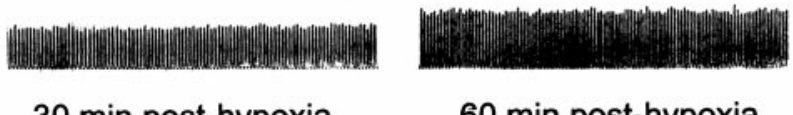

30 min post-hypoxia

60 min post-hypoxia

Figure 1. Integrated phrenic neurogram illustrating three time-dependent responses to isocapnic hypoxia. The top panel shows baseline phrenic nerve activity followed by the increase in phrenic burst amplitude and frequency that takes place during a 5 min bout of hypoxia. The end of hypoxic stimulation is associated with a depression of phrenic burst frequency (posthypoxia frequency decline) relative to baseline (prehypoxic). The bottom panel shows the increase in phrenic burst amplitude 30 and 60 min after the third hypoxic episode that constitutes long-term facilitation.

\section{Short-term hypoxic ventilatory response}

Exposure to hypoxia increased phrenic burst amplitude, expressed as percentage change from prestimulus baseline values (Fig. 4). The burst amplitude was significantly greater than corresponding data from the hyperoxic control group at the same time points. Changes in phrenic burst amplitude during hypoxia were comparable for all four groups (Fig. 4), indicating that neither CDR nor CDR plus ketanserin affected the short-term hypoxic phrenic response.

Potential normalization artifacts caused by variable baseline nerve activities were minimized by also reporting nerve burst amplitude as a change from baseline, expressed as a percentage of the maximal response measured during hypercapnia (percent maximum). Because all results were quantitatively similar, regardless of the normalization used, only data expressed as percent change from baseline will be presented.

Hypoxia also augmented phrenic burst frequency in CDR, CDR plus ketanserin, sham-operated, and hypoxic control rats versus their respective baseline values, and the corresponding value from the hyperoxic control group. However, phrenic burst frequency responses to hypoxia were comparable for all four groups (Fig. $5 A$ ).

\section{Posthypoxia frequency decline}

The period immediately after the first 5 min hypoxic episode was characterized by a depression of respiratory burst frequency that was sustained for the duration of the posthypoxic interval (5 min). The decrease in burst frequency measured $5 \mathrm{~min}$ after hypoxia in the hypoxic control group $(-14 \pm 3$ bursts $/ \mathrm{min}$; relative to the prehypoxic baseline value) was significantly greater than in the hyperoxic control group ( $+2 \pm 4$ bursts/min). This posthypoxia frequency decline was also observed in sham-operated $(-20 \pm 4$ bursts/min at $5 \mathrm{~min}$ after hypoxia), CDR $(-17 \pm 3$ bursts/min at 5 min after hypoxia), and CDR plus ketanserin rats $(-9 \pm 6$ bursts/min at $5 \mathrm{~min}$ after hypoxia). There were no significant differences between any of these hypoxic groups. Some rats displayed a marked decrease in arterial blood pressure during hypoxia with a sharp return toward prehypoxic values on return to hyperoxia; however, there was no consistent trend in the relationship between changes in arterial blood pressure and the changes in respiratory burst frequency observed among the different groups. Thus, changes in arterial blood pressure do not appear to be responsible for posthypoxia frequency decline.
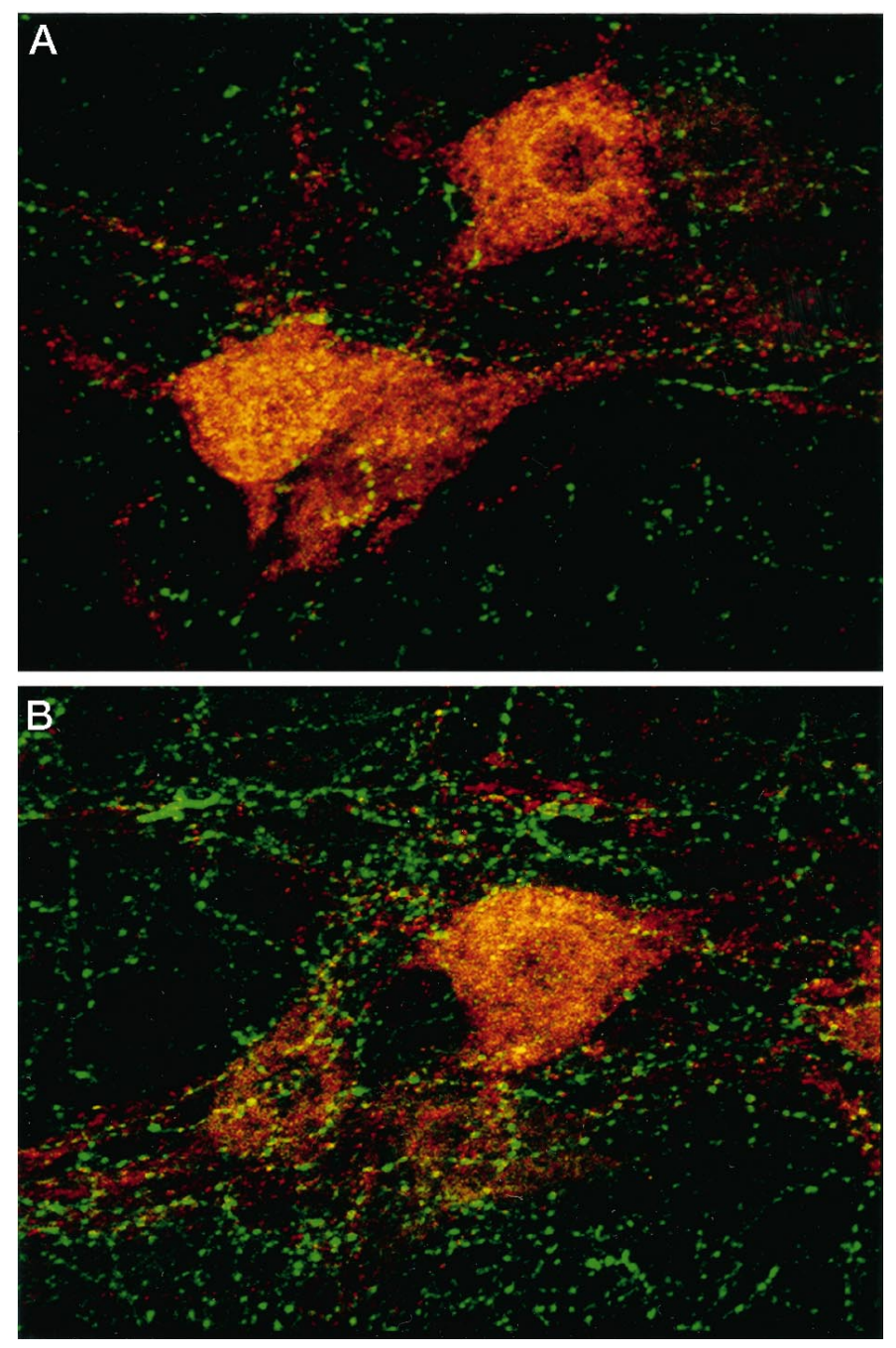

Figure 2. Photomicrographs illustrating serotonergic innervation of cholera toxin-labeled phrenic motor neurons (red), $28 \mathrm{~d}$ after sham surgery $(A)$ or bilateral CDR $(B)\left(\mathrm{C}_{3}-\mathrm{C}_{5}\right)$. These phrenic motoneurons were visualized in the $\mathrm{C}_{4}$ segment using $\mathrm{Cy} 3$-conjugated secondary antibody; Cy5-conjugated secondary antibody was used to visualize 5-HT immunoreactivity. Magnification, $500 \times$. 


\begin{abstract}
Soma

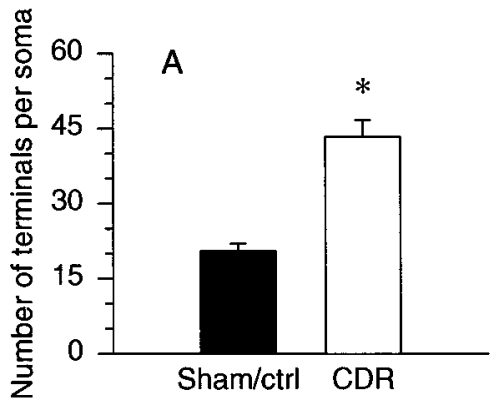

Dendrites

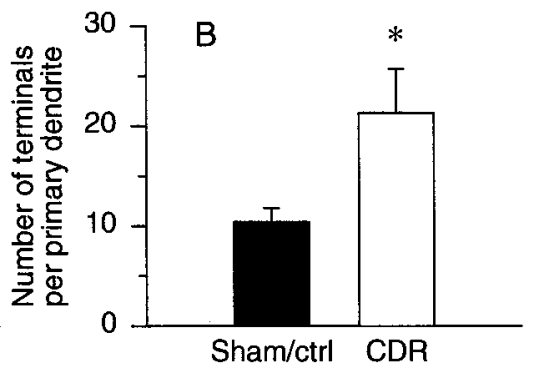

Figure 3. Chronic (28 d) bilateral CDR $\left(\mathrm{C}_{3}-\mathrm{C}_{5}\right)$ increases the number of serotonin-immunoreactive terminals in the immediate vicinity of phrenic motoneuron somas $(A)$ and primary dendrites $(B)$ [CDR, white bars, $n=6$; nondeafferented rats (Sham/ctrl), black bars, $n=6]$. Only terminals located within 5 $\mu \mathrm{m}$ of retrogradely labeled phrenic motoneurons were counted. Because the data obtained from two nonoperated control rats were not significantly different from the sham-operated animals, data were pooled. ${ }^{*} p<0.05$ indicates a value significantly different from the sham-operated/control animals.

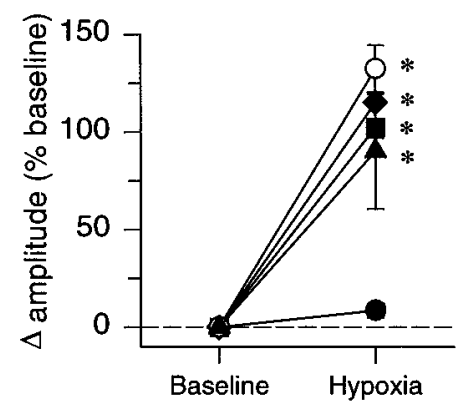

experimental groups other than in rats pretreated with ketanserin. Ketanserin reduced mean arterial blood pressure from $119 \pm 8.5 \mathrm{mmHg}$ to $86 \pm 6.3 \mathrm{mmHg}$ in CDR rats. Although blood pressure of the CDR plus ketanserin group was lower than in the other groups, the time-dependent change between baseline and 60 min after hypoxia $(-14 \pm 12 \%)$ was not different from the average change observed in the other four groups $(-19 \pm 2 \%)$. Thus, it is not likely that ketanserin had indirect effects on long-term facilitation of phrenic motor output via effects on arterial blood pressure.
\end{abstract}

Figure 4. Phrenic burst amplitude response to hypoxia. Data are from the first hypoxic episode in chronically deafferented $(\bigcirc, \mathrm{CDR} ; n=6)$, sham-operated $(\bullet, n=6)$, chronically deafferented and pretreated with ketanserin $(\boldsymbol{\Lambda}, n=6)$, and control $(\boldsymbol{\square}, n=13)$ rats; all data expressed as percent change from baseline. The closed circles represent hyperoxic control rats $(\mathbf{0}: n=7)$ that were not exposed to hypoxia. ${ }^{*} p<0.05$ indicates a value significantly different from the hyperoxic control group.

\section{Long-term facilitation}

After episodic hypoxia, phrenic burst amplitude increased progressively above baseline (i.e., there was long-term facilitation, Fig. 6A). Increases in phrenic burst amplitude $60 \mathrm{~min}$ after hypoxia in $\mathrm{CDR}$, sham, and hypoxic control rats were all greater than in the hyperoxic control rats (Fig. 6A). ANOVA confirmed that burst amplitude progressively increased with time after hypoxia $(p=0.018)$; LTF at 30 min was less than LTF at $60 \mathrm{~min}$ in all three groups (Fig. $6 A$ ).

One hour after hypoxia, long-term facilitation of phrenic burst amplitude was significantly greater in CDR rats $(77 \pm 11 \%)$ versus hypoxic control $(37 \pm 6 \%)$ or sham-operated rats $(29 \pm$ $11 \%$ ) (Fig. 6A). The lack of significant difference between the facilitation recorded from the sham-operated and control groups suggests that surgery per se had no effect on long-term facilitation.

After pretreatment of CDR rats with ketanserin, phrenic burst amplitude decreased below baseline after the last hypoxic episode, and it remained depressed for the duration of the study. One hour after hypoxia, phrenic burst amplitude was significantly lower $(-28 \pm 13 \% ; p<0.05)$ relative to all other rat groups (Fig. 6B).

Although phrenic burst frequency changed over time $(p<$ $0.001)$, there was no significant treatment effect $(p=0.25)$. Thus, the tendency for frequency to increase in these experiments was not significantly greater than the frequency variability inherent to this preparation, as indicated by the hyperoxic control group.

Arterial blood pressure decreased progressively with time in this experimental preparation $(p<0.001)$. However, there were no significant differences in blood pressure changes between

\section{DISCUSSION}

The results of this study indicate that chronic bilateral cervical dorsal rhizotomy enhances serotonergic innervation of the phrenic motor nucleus and serotonin-dependent long-term facilitation of phrenic motor output. Thus, functional plasticity can result from a change in the capacity for serotonergic neuromodulation. More robust serotonergic modulation of inspiratory motoneurons could help compensate for functional deficits caused by chronic deafferentation or other forms of neural injury.

\section{Enhancement of serotonergic innervation to the phrenic motor nucleus}

The enhancement of serotonergic immunoreactivity in the dorsal horn after chronic dorsal rhizotomy has been described previously (Marlier et al., 1991a; Wang et al., 1991; Zhang et al., 1993). On the other hand, little is known about changes in serotonergic innervation of the ventral horn after chronic deafferentation. Recent studies in our laboratory have shown that thoracic dorsal rhizotomy enhances immunoreactive serotonin terminal density in both the spinal dorsal and ventral horns of goats (Mitchell et al., 1992) and serotonin concentration in distant spinal segments (cervical) (Mitchell et al., 1995; Turner et al., 1997). However, these changes could not be ascribed to more specific targets, such as respiratory motor nuclei. The greater number of serotoninimmunoreactive terminals innervating the phrenic motor nucleus after CDR in rats confirms that the increased serotonergic innervation is specific to an important respiratory motor nucleus, although increased serotonergic innervation may not be unique to this target site.

The mechanisms promoting sprouting of serotoninimmunoreactive terminals in response to chronic deafferentation are not known. It is known, however, that stimulation of chemoreceptor fibers in the carotid sinus nerve increases the discharge rate of raphe neurons (Yates et al., 1992; Morris et al., 1996) and enhances fos-like immunoreactivity in raphe pallidus (Erickson and Millhorn, 1991; Teppema et al., 1997). Because increased c-fos expression (and other immediate-early genes) may play a 
A

Short-term response

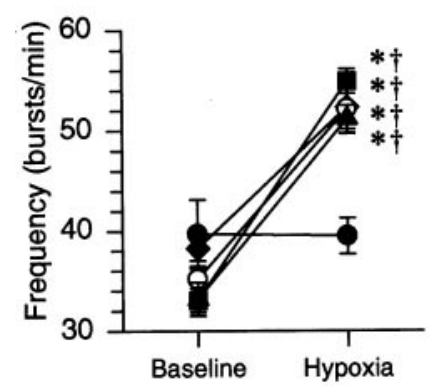

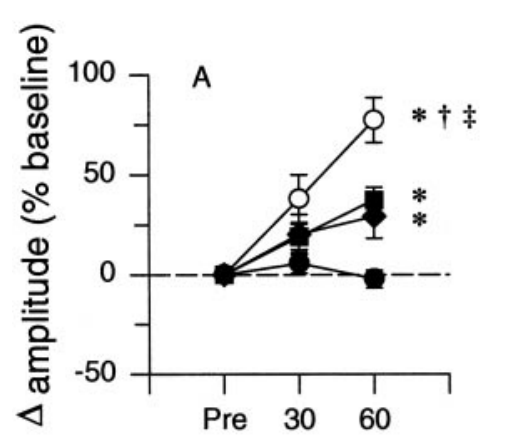

Time post-stimulation (min)
B Long-term facilitation

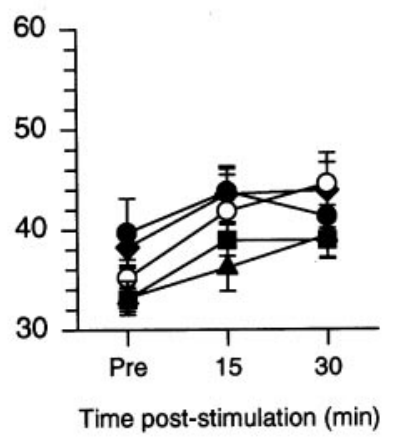

Figure 5. A, Respiratory burst frequency during hypoxia: the short-term-hypoxic response. $B$, Respiratory burst frequency measured before ( pre), 30, and 60 min after the final hypoxic episode, long-term facilitation. $\mathrm{O}, \mathrm{CDR}$; , sham-operated, $\mathbf{\square}$, control, $\boldsymbol{\Delta}$ CDR plus ketanserin, and $\bullet$, hyperoxic control. * $p<0.05$, significantly different from the hyperoxic control group; $\dagger$ significantly different from baseline.

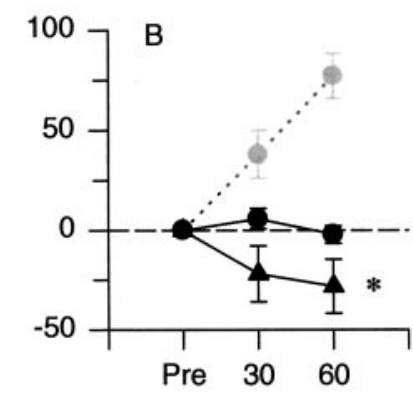

Time post-stimulation (min)
Figure 6. A, Long-term facilitation of phrenic burst amplitude is enhanced in chronically deafferented $(\bigcirc, \mathrm{CDR} ; n=6)$ rats relative to sham-operated $(\forall, n=6)$ and control $(\mathbf{\square}, n=$ 13) rats. The closed circles represent hyperoxic (time) control rats $(\bullet, n=7)$ that were not exposed to hypoxia. $B$, Reversal of long-term facilitation in CDR rats pretreated with the $5-\mathrm{HT}_{2}$ receptor antagonist ketanserin $(\bullet, \mathrm{CDR}$ plus ketanserin; $n=6$ ). Integrated phrenic burst amplitude is expressed as percent baseline at 30 and $60 \mathrm{~min}$ after the last hypoxic episode. ${ }^{*} p<0.05$, significantly different from the hyperoxic control group; $\dagger p<0.05$, significantly different from shamoperated group; $\ddagger p<0.05$, significantly different from the hypoxic control group. role in the coupling between short-term events and long-term changes in gene expression (Morgan and Curran, 1989), it is possible that an activity-dependent mechanism underlies the reconfiguration of serotonergic projections. In this hypothetical scheme, the increase in raphe neuron activity required for enhancement of descending serotonergic innervation could result from: (1) a functional deficit in respiratory motor output leading to intermittent hypoxia after dorsal rhizotomy or (2) the removal of inhibitory synaptic inputs to raphe neurons, leading to disinhibition after dorsal rhizotomy. Although there is some evidence for acute deficits in respiratory motor function after cervical dorsal rhizotomy (Nathan and Sears, 1960; Forster et al., 1994), the potential relationship between a deficit in respiratory motor function and enhancement of serotonergic innervation of respiratory motor nuclei remains to be tested. It is also possible that the changes in serotonergic innervation of the phrenic motor nucleus are related to changes in the expression of spinal neurotrophic factors, indirectly affecting raphe neurons.

Chronic CDR elicits numerous complex changes within the CNS that may affect respiratory motor function. For instance, the number of radio-ligand-labeled $5-\mathrm{HT}_{1 \mathrm{~A}}, 5-\mathrm{HT}_{1 \mathrm{~B}}$, and $5-\mathrm{HT}_{3}$ receptor subtypes is significantly reduced in the dorsal horn $10 \mathrm{~d}$ after unilateral dorsal rhizotomy $\left(\mathrm{C}_{4}-\mathrm{T}_{2}\right)$ in rats (Laporte et al., 1995). Each of these receptor subtypes is associated with presynaptic or postsynaptic inhibition of primary afferent inputs in the spinal dorsal horn. However, long-term facilitation more clearly requires the activation of 5- $\mathrm{HT}_{2}$ receptors (Kinkead and Mitchell, unpublished observations), which are more likely located in the ventral horn (Marlier et al., 1991b). The effects of dorsal rhizotomy on this receptor subtype are unknown.

\section{Functional significance of changes in serotonergic innervation}

Short-term hypoxic responses

Short-term phrenic responses to hypoxia were unaffected by CDR, with or without ketanserin pretreatment. This suggests that either: (1) CDR had no effect on this response or (2) that the changes in serotonergic innervation may have offset the potential effects of CDR on this response by activating 5-HT receptors that are unaffected by ketanserin.

\section{Posthypoxia frequency decline}

PHFD has been described as a form of "memory" within the respiratory control system (Hayashi et al., 1993; Powell et al., 1998). The mechanisms underpinning PHFD are not clear. However, it has been postulated that $\alpha_{2}$-adrenoceptor activation contributes to the decline in respiratory frequency after hypoxia (Bach and Mitchell, 1996b). Recent studies suggest that 5-HT receptor activation may also modulate this behavior (Kinkead and Mitchell, 1998). Based on the latter observation, we predicted that CDR and/or CDR with ketanserin may affect PHFD. Nevertheless, the present data do not support this hypothesis and suggest that the enhancement of descending serotonergic innervation attendant to $\mathrm{CDR}$ had no effect on respiratory rhythmogenesis.

\section{Long-term facilitation after $C D R$}

Long-term facilitation is a form of activity-dependent plasticity or "memory" in respiratory motor function which requires serotonin receptor activation for its manifestation (Millhorn et al., 1980b; Hayashi et al., 1993; Fregosi and Mitchell, 1994; McCrimmon et al., 1995; Bach and Mitchell, 1996a). Bach and Mitchell 
(1996a) proposed that serotonin acts on $5-\mathrm{HT}_{2}$ receptor subtypes at the level of the respiratory motor nucleus. In support of this hypothesis, pretreatment of rats with the selective $5-\mathrm{HT}_{2}$ receptor antagonist ketanserin prevents long-term facilitation of phrenic motor output (Kinkead and Mitchell, unpublished observations). The observation that ketanserin actually reverses LTF, leading to respiratory depression in CDR rats suggests that the enhancement of long-term facilitation of phrenic motor output in CDR rats is caused by an amplification of the same serotonindependent mechanism and that it is directly related to more robust serotonergic innervation (and modulation) of the phrenic motoneuron pool. On the other hand, ketanserin pretreatment blocks long-term facilitation without causing a long-lasting depression of phrenic motor output in control rats (Kinkead and Mitchell, unpublished observations). Although the mechanism or mechanisms mediating this long-lasting depression of respiratory motor output in ketanserin-treated CDR rats are unknown, the response is similar to an $\alpha_{2}$-dependent long-term depression of respiratory activity after episodic hypercapnia (Bach and Mitchell, 1998). This observation raises the possibility that CDR elicits plasticity of other monoaminergic pathways that exert long-lasting modulatory effects on phrenic motor output after episodic hypoxia (e.g., noradrenergic neurons).

The degree of long-term facilitation of phrenic motor output reported in this paper is blunted in comparison to a previous report from our laboratory (Bach and Mitchell, 1996a). Specifically, the increase in phrenic burst amplitude during long-term facilitation $(37 \pm 6 \%)$ was less than that reported by Bach and Mitchell (1996a) $(63 \pm 17 \% ; p<0.05)$. Furthermore, in the present study, none of the groups showed a significant increase of burst frequency after episodic hypoxia, contrasting with our previous report. One potential explanation for these differences is that each study was done on different substrains of Sprague Dawley rats. Bach and Mitchell (1996a) used Sprague Dawley rats obtained from Sasco (Madison, WI), whereas Sprague Dawley rats obtained from Harlan (Madison, WI) were used in the present study. These substrains of rats differ in the pattern of noradreneregic innervation of the spinal cord (Clark and Proudfit, 1992; Sluka and Westlund, 1992), raising the possibility that other anatomical and, perhaps, functional differences related to monoaminergic systems exist between Harlan and Sasco Sprague Dawley rats.

\section{Significance}

Our results suggest that plasticity of descending serotonergic innervation of phrenic motoneurons elicited by chronic deafferentation mediates functional plasticity of respiratory motor output. The enhancement of long-term facilitation of inspiratory motor output in CDR rats therefore constitutes an example of functional plasticity caused by a greater capacity for serotonergic neuromodulation. The capacity for functional plasticity via changes in neuromodulatory systems may be of considerable significance to functional recovery after a neural injury or as a compensatory mechanism for functional deficits caused by the onset of (respiratory) disease.

\section{REFERENCES}

Azmitia EC, Whitaker-Azmitia PM (1991) Awakening the sleeping giant: anatomy and plasticity of the brain serotonergic system. J Clin Psychiatry 52:4-16.

Bach KB, Mitchell GS (1996a) Hypoxia-induced long-term facilitation of respiratory activity is serotonin dependent. Respir Physiol 104:251-260.
Bach KB, Mitchell GS (1996b) Post hypoxic depression (PHD) of phrenic burst frequency in rats requires $\alpha_{2}$ adrenergic receptors. Physiologist 39:185.

Bach KB, Mitchell GS (1998) Hypercapnia induced long term depression of respiratory activity requires $\alpha_{2}$-adrenergic receptors. J Appl Physiol 84:2099-2105.

Clark FM, Proudfit HK (1992) Anatomical evidence for genetic differences in the innervation of the rat spinal cord by noradrenergic locus coeruleus neurons. Brain Res 591:44-53.

Coles SK, Dick TE (1996) Neurones in the ventrolateral pons are required for post-hypoxic frequency decline in rats. J Physiol (Lond) 497:79-94.

Eldridge FL, Millhorn DE (1986) Oscillation, gating, and memory in the respiratory control system. In: Handbook of physiology: the respiratory system (Fishman AP, ed), pp 93-114. Washington, DC: American Physiological Society.

Erickson JT, Millhorn DE (1991) Fos-like protein is induced in neurons of the medulla oblongata after stimulation of the carotid sinus nerve in awake and anesthetized rats. Brain Res 567:11-24.

Forster HV, Lowry TF, Pan LG, Erickson BK, Korucki MJ, Forster MA (1994) Diaphragm and lung afferents contribute to inspiratory load compensation in awake ponies. J Appl Physiol 76:1330-1339.

Fregosi RF, Mitchell GS (1994) Long-term facilitation of inspiratory intercostal nerve activity following repeated carotid sinus nerve stimulation in cats. J Physiol (Lond) 477.3:469-479.

Hayashi F, Coles SK, Bach KB, Mitchell GS, McCrimmon DR (1993) Time dependent phrenic nerve responses to carotid afferent activation: intact vs decerebellate rats. Am J Physiol 265:R811-R819.

Kinkead R, Mitchell GS (1998) Serotonin modulates post-hypoxia frequency decline (PHFD) in rats. FASEB J 12:A780.

Kinkead R, Zhan WZ, Prakash YS, Bach KB, Sieck GC, Mitchell GS (1997) Cervical dorsal rhizotomy (CDR) enhances long-term facilitation of respiratory motor output in rats. FASEB J 11:A207.

Laporte A, Fattaccini C, Lombard M, Chauveau J, Hamon M (1995) Effects of dorsal rhizotomy and selective lesion of serotonergic and noradrenergic systems on $5-\mathrm{HT}_{1 \mathrm{a}}, 5-\mathrm{HT}_{1 \mathrm{~b}}$ and $5-\mathrm{HT}_{3}$ receptors in rat spinal cord. J Neural Transm 100:207-223.

Marlier L, Poulat P, Rafjaofetra N, Privat A (1991a) Modification of serotonergic immunoreactive pattern in the dorsal horn of the rat spinal cord following dorsal root rhizotomy. Neurosci Lett 128:9-12.

Marlier L, Teilhac JR, Cerruti C, Privat A (1991b) Autoradiographic mapping of $5 \mathrm{HT}_{1 \mathrm{~A}}, 5-\mathrm{HT}_{1 \mathrm{~B}}$ and $5-\mathrm{HT}_{2}$ receptors in the rats spinal cord. Brain Res 550:15-23.

McCrimmon DR, Dekin MS, Mitchell GS (1995) Glutamate, GABA, and serotonin in ventilatory control. In: Regulation of Breathing (Dempsey JA, Pack AI, eds), pp 151-218. New York: Marcel Dekker.

Millhorn DE, Eldridge FL, Waldrop TG (1980a) Prolonged stimulation of respiration by a new central neural mechanism. Respir Physiol 41:87-103.

Millhorn DE, Eldridge FL, Waldrop TG (1980b) Prolonged stimulation of respiration by endogenous central serotonin. Respir Physiol 42:171-198.

Mitchell GS, Sloan HE, Foley KT, Brownfield MS, Miletic V (1992) Increased serotonin in the thoracic spinal cord of goats following chronic thoracic dorsal rhizotomy (TDR). FASEB J 6:A1507.

Mitchell, GS, Foley KT, Martin PA, Olson EB, Miletic V, Brownfield, MS (1995) Increased spinal monoamine concentrations following thoracic dorsal rhizotomy (TDR) in goats. Soc Neurosci Abstr 21:p1886.

Morgan JL, Curran T (1989) Stimulus-transcription coupling in neurons: role of cellular immediate-early genes. Trends Neurosci 12:459-462.

Morris KF, Arata A, Shannon R, Lindsey BG (1996) Long-term facilitation of phrenic nerve activity in cats: responses and short time scale correlations of medullary neurones. J Physiol (Lond) 490.2:463-480.

Nathan PW, Sears TA (1960) Effects of posterior root section on the activity of some muscles in man. J Neurol Neurosurg Psychiatry 23:10-22.

Powell FL, Milsom WK, Mitchell GS (1998) Time domains of the hypoxic ventilatory response. Respir Physiol 112:123-134.

Prakash YS, Smithson KG, Sieck, GC (1993) Measurements of motoneuron somal volumes using laser confocal microscopy: Comparisons with shape-based stereological estimations. NeuroImage 1:95-107.

Prakash YS, Kinkead R, Zhan YS, Smithson KG, Zhan P, Sieck GC, Mitchell GS (1997) Serotonergic innervation of phrenic motoneurons is increased by cervical dorsal rhizotomy. FASEB J 11:A206. 
Sluka KA, Westlund KN (1992) Spinal projections of the locus coeruleus and the nucleus subcoeruleus in the Harlan and the Sasco Sprague-Dawley rat. Brain Res 579:67-73.

Teppema LJ, Veening JG, Kranenburg A, Dahan A, Berkenbosch A, Olievier C (1997) Expression of $c$-fos in the rat brainstem after exposure to hypoxia and normoxic and hyperoxic hypercapnia. J Comp Neurol 388:169-190.

Turner DL, Bach KB, Martin PA, Olsen EB, Brownfield M, Foley KT, Mitchell GS (1997) Modulation of ventilatory control during exercise. Respir Physiol 110:277-285.

Wang SD, Goldberger ME, Murray M (1991) Plasticity of spinal systems after unilateral lumbosacral dorsal rhizotomy in the adult rat. J Comp Neurol 304:555-568.
Whitaker-Azmitia PM, Azmitia EC (1991) Serotonin trophic factors in development, plasticity and aging. In: Serotonin: molecular biology, receptors and functional effects (Fozard JR, Saxena PR, eds), pp 43-49. Basel: Birkhauser Verlag.

Yates BJ, Goto T, Bolton PS (1992) Responses of neurons in the caudal medullary raphe nuclei of the cat to stimulation of the vestibular nerve. Exp Brain Res 89:323-332.

Zifa E, Fillion G (1992) 5-Hydroxytryptamine receptors. Pharmacol Rev 44:401-458.

Zhang B, Goldberger ME, Murray M (1993) Proliferation of SP- and 5HT-containing terminals in lamina II of rat spinal cord following dorsal rhizotomy: quantitative EM-immunocytochemical studies. Exp Neurol 123:51-63. 